\title{
Inhomogeneous Hardness Distribution of High Pressure Torsion Processed IF Steel Disks
}

\author{
Yuepeng Song ${ }^{1,2,3^{*}}$, Wenke Wang ${ }^{1,3}$, Dongsheng Gao ${ }^{3}$, Hyoung-Seop Kim ${ }^{2 *}$, Eun-Yoo Yoon ${ }^{2}$, \\ Dong-Jun Lee ${ }^{2}$, Chong-Soo Lee ${ }^{2}$, Jing Guo ${ }^{1,3}$
}

${ }^{1}$ Mechanical and Electronic Engineering College, Shandong Agricultural University, Tai'an, China; ${ }^{2}$ Department of Materials Science and Engineering, Pohang University of Science and Technology, Pohang, Korea; ${ }^{3}$ Shandong Provincial Key Laboratory of Horticultural Machineries and Equipments, Shandong Agricultural University, Tai'an, China.

Email: " ustbsong@sina.com, ${ }^{*}$ hyoungseopkim@gmail.com

Received November $7^{\text {th }}, 2011$; revised December $24^{\text {th }}, 2011$; accepted February $6^{\text {th }}, 2012$

\begin{abstract}
The inhomogeneous hardness distribution of high pressure torsion (HPT) processed IF steel disks along different directions is investigated. The results indicated that there exists inhomogeneous distribution in HPT processed IF steel disks, giving lower hardness in the center and higher hardness in the edge regions. However, on the axisymmetrical section testing plane of the disks' thickness direction, there is a soft zone near the surface of disks. Further results from radius testing plane of different depths from the surface of HPT processed disks show that the inhomogeneity rules of hardness distribution on the radius direction are similar to that on the thickness direction. Compared with the initial state, different stages of HPT (compression and compression + torsion) can both remarkably increase the hardness of IF steel disks. Microstructure investigation results can give a well support to verify the rules of hardness distribution, showing hardly no change of grains in center and sever plastic deformation in edge. The inhomogeneous distribution of stress and strain with the huge friction between anvil and disks in the process of HPT play an important role of hardness and microstructure distribution.
\end{abstract}

Keywords: Hardness Distribution; High-Pressure Torsion (HPT); IF Steel; Inhomogeneous

\section{Introduction}

The processing of bulk metals through the application of High Pressure Torsion (HPT) is considered to be the most successful procedure for producing ultrafine-grained (UFG) materials with exceptionally small grain sizes on submicrometer or nanometer level, and with a high fraction of boundaries having high angles of misorientation [1-3]. At present, in a wide range of pure metals, metallic alloys and intermetallics [1-7], bulk solids with UFG- or nano-microstructures have been produced by using HPT technique. Because of high strength, superior fatigue and a potential super-plastic forming capability at elevated temperatures, they are attractive candidate materials for parts in the automotive and aerospace sections $[5,8]$. Interstitial-free (IF) steel is widely used for automotive deep-drawing applications with micro-alloying elements $(\mathrm{Ti}, \mathrm{Nb}$ and $\mathrm{V}$ ) forming precipitates with carbon and nitrogen. Because of the absence of interstitial solutes and the appropriate texture in IF steel, an excellent deepdrawability will proceed. The investigation of severe

\footnotetext{
*Corresponding authors.
}

plastic deformation (SPD) like equal-channel angular pressing (ECAP) of IF steel has being attracted considerable interest of researchers and demonstrated a practical applicability of SPD for improving its mechanical properties $[9,10]$. However, until now, there is no research on the HPT processed IF steel disks, as far as the authors know.

There is a significant limitation with HPT because of the inhomogeneous strain, at least in principle, across the diameter of the samples during the HPT deformation, which can lead to the homogeneity of hardness and microstructure of disks and be verified by materials such as $\mathrm{Al}, \mathrm{Ni}, \mathrm{Cu}$ and austenitic steel for giving the lower values of hardness in the centers but higher hardness in the edge of disks $[1,5]$. However, recently experiment results of pure Al material of HPT indicated that there are higher hardness values in the centers of the disks [11,12]. The study on inhomogeneity of HPT processed IF steel disks is particularly concerned by materials researchers.

In this paper, we investigate the inhomogeneity of hardness and microstructure distribution on the different direction of IF steel disks processed by HPT. 


\section{Experimental Procedures}

The investigating IF steel was manufactured by the Pohang Steel Company (POSCO, Korea) with the composition of $0.0026 \mathrm{wt} \% \mathrm{C}, 0.096 \mathrm{wt} \% \mathrm{Mn}, 0.045 \mathrm{wt} \% \mathrm{Al}$ and $0.041 \mathrm{wt} \% \mathrm{Ti}$. The initial state of material is a plate 12 $\mathrm{mm}$ in thickness size rolled from casting ingot. After homogenizing (973 K $\times 2 \mathrm{~h}$, cooling in furnace), the grain size is $150-200 \mu \mathrm{m}$ and hardness is about $80 \mathrm{Hv}$.

For the experiments, application pressure of $6 \mathrm{GPa}$ was imposed at room temperature on the disks. The time of compression load is set as 10 seconds. Meanwhile, two revolutions of 0 and 1 turns are conducted, the samples namely IF60 (no turn) and IF61 (1 turn), respectively. The axial profiles of HPT disks are ellipse, with $10 \mathrm{~mm}$ of major axis and $2 \mathrm{~mm}$ of minor axis.

There are two directions of hardness distribution measurement: along the longitudinal direction (namely LD position) and transversal direction (namely TD position). On the LD position, the hardness testing plane is the axisymmetrical section of HPT disks. Equal-distance testing points with $0.5 \mathrm{~mm}$ on the testing lines follows the radius direction on the axisymmetrical section plane of HPT disks. While on TD position, the mounting samples were grinded and polished layer by layer and the hardness testing planes were so obtained with different depth from surface. The schematic drawing of HPT disks and the measurement of hardness distribution on different direction is shown as Figure 1.

Hardness was measured using FM-700 Microhardness Tester and the pressure loading is $100 \mathrm{~g}$, continuous $10 \mathrm{~s}$. The color-coded contour maps and curves of hardness distribution of different samples are so obtained. Microstructures in different position of disks were observed using optical microscopy (Olympus U-TV0.5xc) taken from the discs after HPT.

\section{Experimental Results and Discussion}

\subsection{Inhomogeneous Hardness Distribution on the Thickness Direction of HPT Disks}

Until now, no systematic works of hardness and micro- structure distribution on the LD position of HPT disks at the compressive stage has been done, as far as the authors know. Figure 2 shows the hardness distribution on the axisymmetrical section testing plane of HPT disks.

As the same rules with lots of literatures [5], the figures clearly indicated lower values of hardness in the center and higher values in the edge. i.e. as to the central lines on the testing plane, the hardness in the edge, middle and center is $190.1 \mathrm{Hv}, 155.1 \mathrm{Hv}$ and $153 \mathrm{Hv}$ for IF60 (only compression) and $379.5 \mathrm{Hv}, 330.7 \mathrm{Hv}$ and $249.3 \mathrm{Hv}$ for IF61 (1 turn), respectively. Compared with the initial state $(80 \mathrm{Hv})$, the HPT processing can increase the hardness of disks remarkably, especially the effect of torsion on hardness. For this study, one turn of HPT can increase the hardness above 3 times in the edge of disks. Hence, there also exists an inhomogeneity, for IF steel materials, across the center to edge of HPT disks, not only in compressive stage but also torsion stage.

The hardness distribution color-coded contour maps of different samples on the LD position can clearly indicate this rule as shown in Figure 3. It must be pointed out that the hardness scale of the two contour maps is different because of their large difference.

From these figures, almost symmetrical distribution of hardness exists between top and bottom section of HPT disks with the central plane. Furthermore, a low hardness region exits in the center near the surface of disks is also clearly displayed.

\subsection{Hardness Distribution on the TD Position}

On the TD position, the testing planes of different distance from surface of HPT disks were obtained by grinding and polishing the mounting samples layer by layer. Hardness distribution on testing planes of different depth from surface is displayed in the Figure 4.

From Figure 4, the inhomogeneous hardness distribution on the transversal direction is similar to that of on the longitudinal direction, giving lower hardness in the center but higher values in the peripheral regions of HPT disks. Further results indicated that compared with compressive stage, torsion can make the variation of hardness

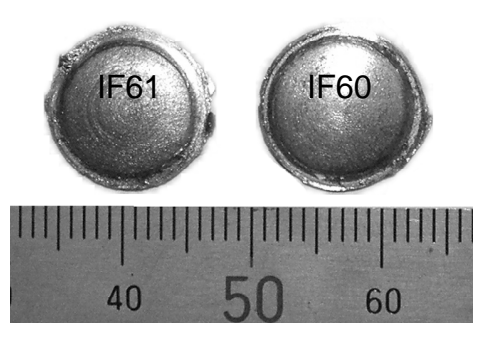

(a)

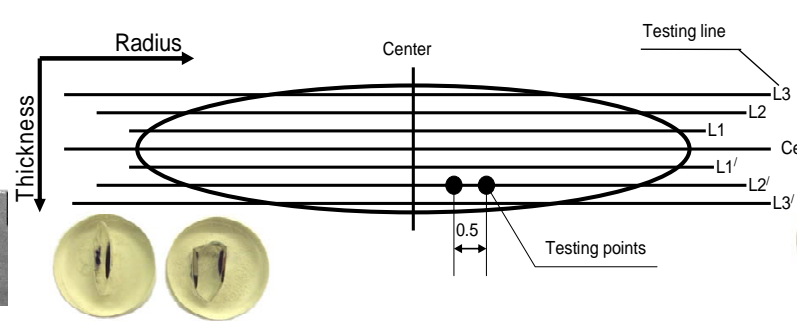

(b)

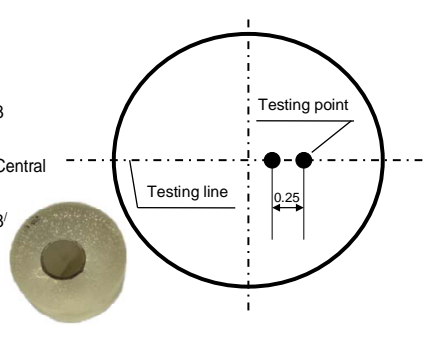

(c)

Figure 1. The schematic drawing of HPT disks and the measurement method. (a) HPT disks; (b) On LD position; (c) On TD position. 


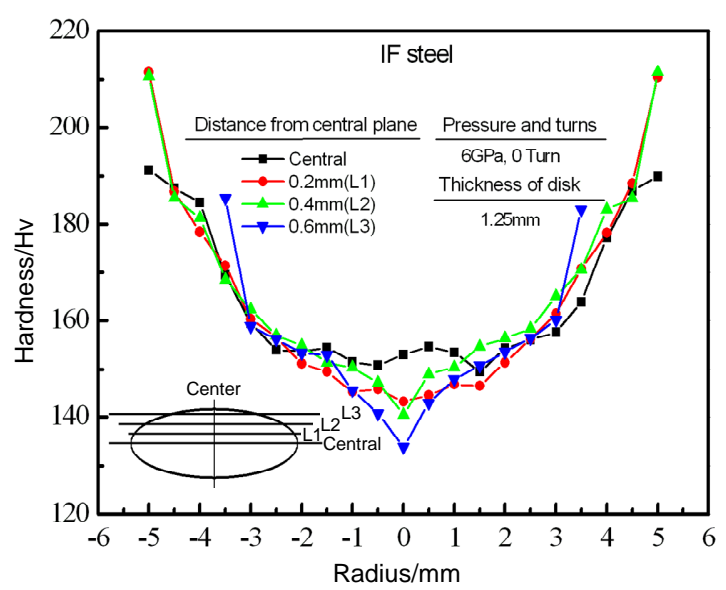

(a)

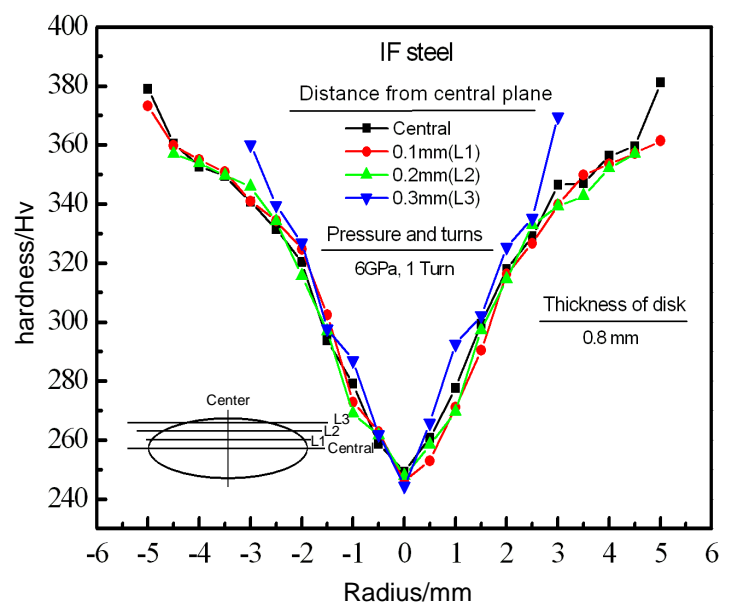

(b)

Figure 2. Hardness distribution of IF steel disks processed by HPT on the thickness direction. (a) Only compression (0 turn); (b) 1 turn.

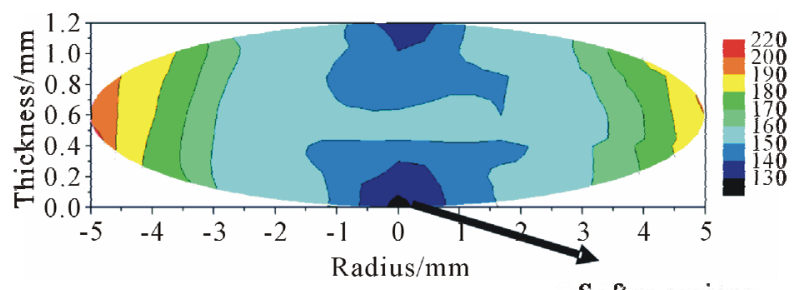

(a)

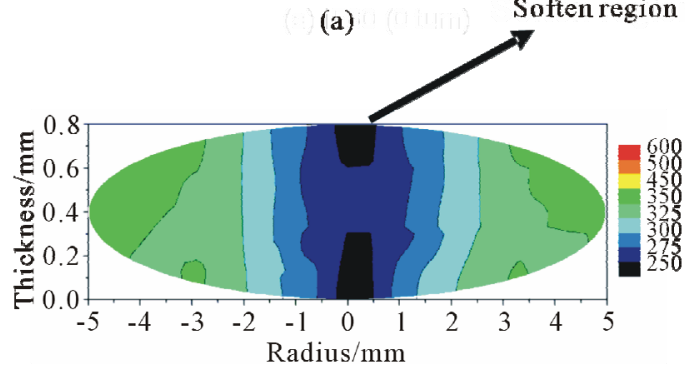

(b)

Figure 3. Hardness distribution color-coded maps of HPT disks on the thickness direction. (a) IF60 (0 turn); (b) IF61 (1 turn).

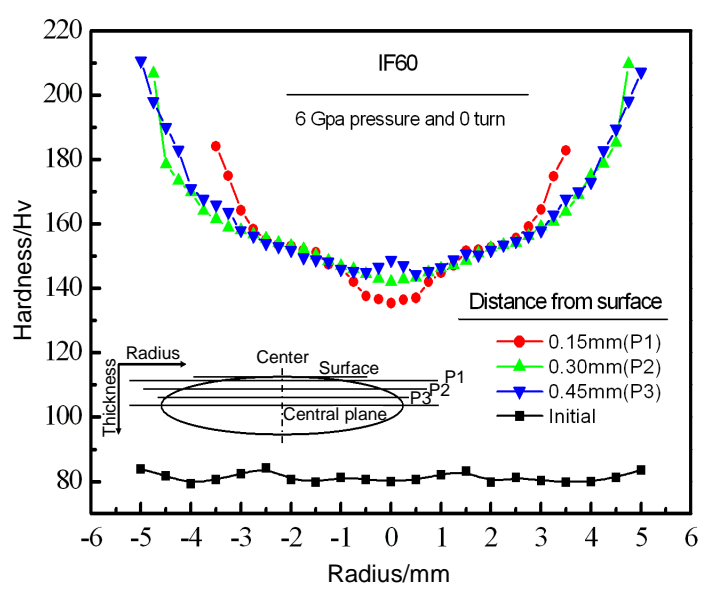

(a)

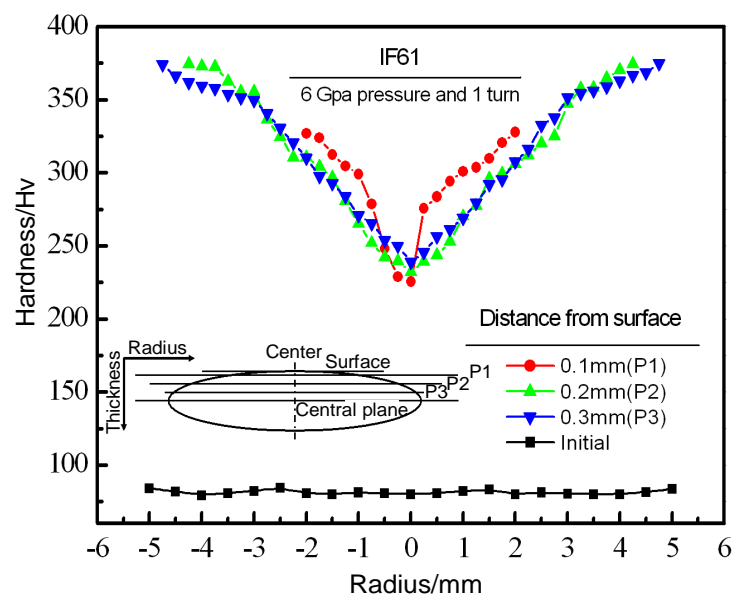

(b)

Figure 4. Hardness distribution on testing planes of different depth from surface.

from center to edge increasing more sharply as shown in Figure 5.

\subsection{Inhomogeneity of Microstructure Distribution of IF Steel Disks by HPT Processed}

Difference in hardness is attributed to the microstructure of different position on HPT processed IF steel disks. As an example, the microstructure of HPT disks on the LD position is shown in Figure 6.

Figure 6 clearly indicates that there is a remarkable inhomogeneity distribution of microstructure on the LD position, for the HPT disks of not only at the compressive stage but also at torsion stage. From the Figure 6, the grains of center have no hardly change as the same as the initial state, however, in the edge, grains occur large severe plastic deformation and their boundaries become very obscure. Especially, for the IF60 sample, the flowline characteristic microstructure as highly deformed is clearly displayed. This severe deformed microstructure is 


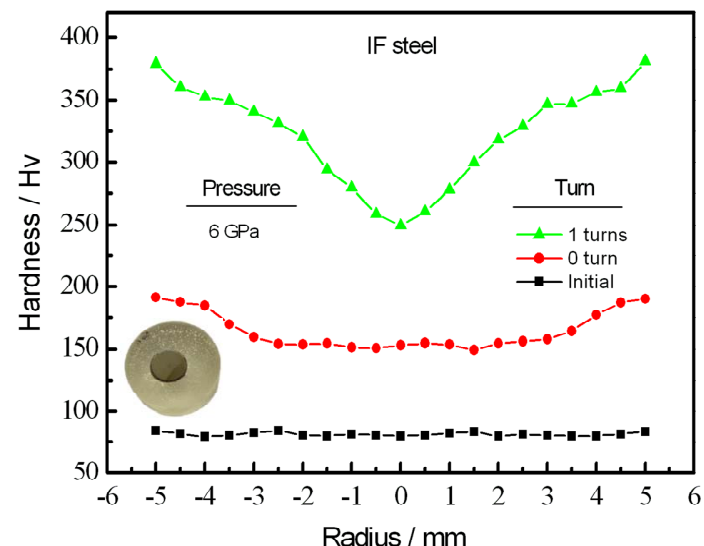

Figure 5. Hardness distribution of HPT IF steel disks on the radius direction.

similar to that observed in a very wide range of ultrafinegrained materials produced through processing by HPT. Further investigation of microstructure on the TD position of HPT disks has the same inhomogeneity distribution rules.

The inhomogeneity distribution of microstructure can support the ones of hardness, giving lower hardness in center and higher hardness in edge. The key factors of inhomogeneity distribution of hardness and microstructure are attributed to the inhomogeneity deformation of HPT disks. That is to say, the inhomogeneous distribu- tion of strain and stress leads to microstructure inhomogeneity. As we know, the mechanical properties of the deformed material are attribution to the amount of plastic deformation, i.e. the development and distribution of strain and stress of disks during the HPT processing [13, 14].

The formula of equivalent von Mises strain $\varepsilon_{e q}$ for estimating the strain on the HPT disks is $[2,5,15]$,

$$
\varepsilon_{e q}=\frac{2 \pi N r}{h \sqrt{3}}
$$

here, $N$ is the amount of turns, $h, r$ is the thickness and distance from center of disks, respectively.

From the formula (1), following the radius direction from center to edge of HPT disks, along the $r$ increasing, the equivalent von Mises strain is higher to higher which means the deformation of material more and more severe. For this study, because of the arc shape of disks for the lower to lower of thickness $h$ from center to edge, the equivalent von Mises strain even more sharply variation and strengthens the effect of hardability on material. So the arc shape of HPT disks leads to the more inhomogeneous strain distribution during deformation. Compared with the disks only in compressive of HPT, the torsion can make the strain more inhomogeneous from center to edge for giving higher strain value in edge and lower strain in center. Correspondingly, more severe plastic

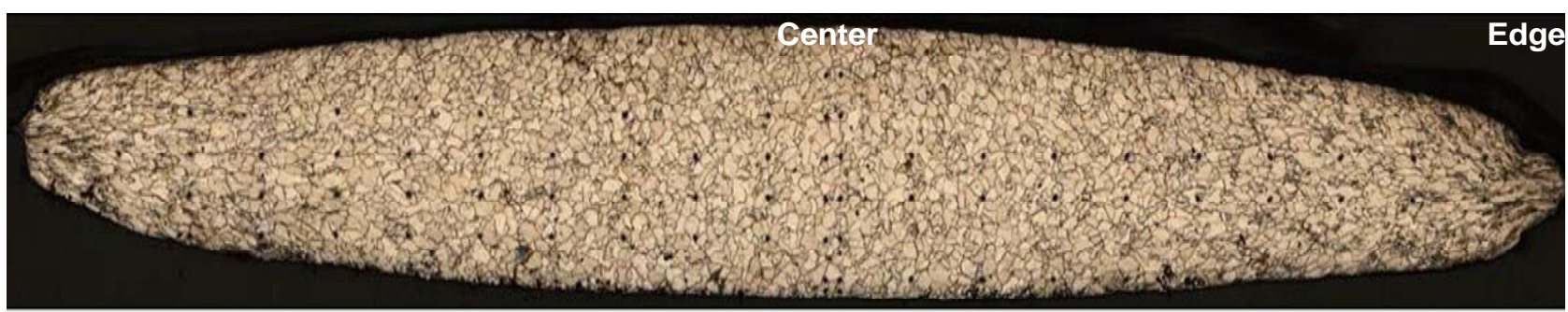

(a)

$1 \mathrm{~mm}$

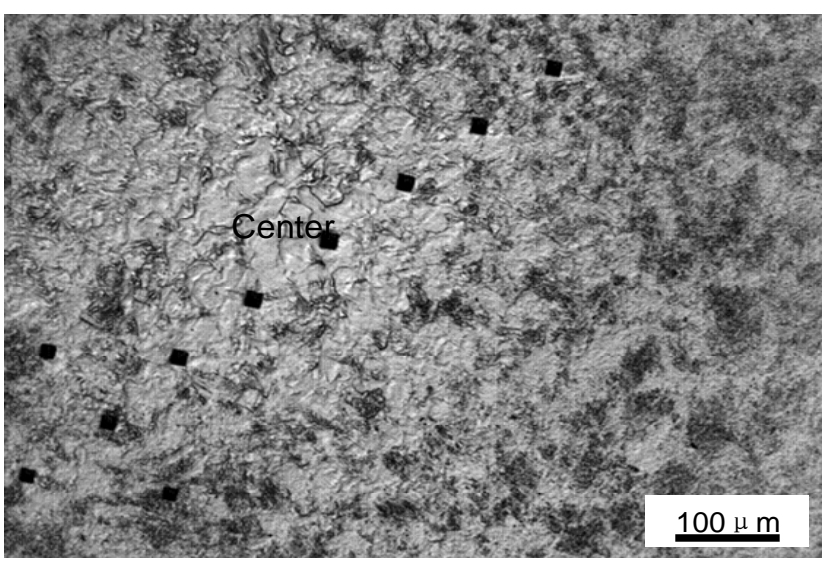

(b)

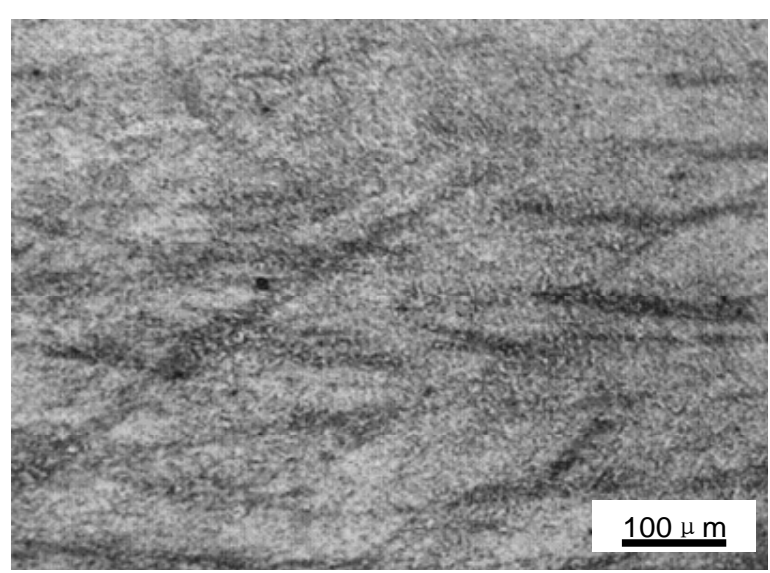

(c)

Figure 6. Microstructure of HPT disks on the LD position. (a) IF60 (0 turn); (b) IF61 (1 turn)—center; (c) IF61 (1 turn)—edge. 
deformation occurs in edge while no changes in center, which is the key reason why the hardness and microstructure display so inhomogeneity of HPT disks not only on the LD position but also on the TD position.

However, because of no turn that means $N$ in formula (1) is zero, as to the disks only at the compressive stage of HPT, the inhomogeneity of hardness and microstructure distribution will not be explained well by formula (1). In fact, the deformation of disks at the compression stage of HPT is similar to that of the upsetting process of forging technology. The friction between the punch and workpiece during the deformation plays an important role. Recently, the important effect of friction on the deformation of materials during the compressive stage is paid attention by many researchers $[4,16,17]$. The results indicated that the strain will be depended on the degree of difficulty in rheology deformation of materials. According to the sample IF60 (only compression), the material in the edge of disks will be easily to rheologically deform into the edge flash where displays the characteristic flow-line microstructure. However, in the center, especially near the surface because of huge friction with groove of anvil, the rheological deformation is difficult to proceed which leads to the lower strain and no hardly change of grains. The soften region near the surface in center of disks only compressed will so exist.

\section{Conclusion}

Inhomogeneous hardness distribution of HPT processed IF steel disks along different directions is studied. According to the disks of not only at compressive stage but also at torsion stage, on the longitudinal direction, there exists an inhomogeneous hardness distribution on the axisymmetrical section testing plane of HPT disks, for giving lower hardness value in center and higher hardness in edge. The inhomogeneity rules of hardness distribution of HPT disks were verified with the testing planes of different depth from surface on the transversal direction. Moreover, a soften region near the surface of disks is investigated. Compared with the initial state, different stages of HPT (compression and compression + torsion) can both remarkably increase the hardness of IF steel disks. For this study, the torsion makes the variation of hardness from center to edge more sharply. The microstructure observation was found to correlate well with the hardness distribution corresponding to the strain of different position of HPT disks. At the compressive stage of HPT, the strain will be depend on the degree of difficulty in rheology deformation, however for torsion stage, on the thickness and distance from center to edge direction. Especially, the friction between the punch and workpiece during the deformation plays an important role.

\section{Acknowledgements}

Y. P. Song acknowledges the postdoctoral fellowship supported by the National Research Foundation through the Korea-China Young Scientists Program, Korea. The HPT samples were provided by Prof. Hyoungseop Kim of POSTECH, Korea. Furthermore, this work also was supported by Key Laboratory of Functional Crystals and Laser Technology, TIPC, CAS, Graduate Innovative program of Shandong Province (SDYY11079), NSFC (5100 1111), Program of "Twelfth Five-Year" National Science and Technology Support Plan (2011BAD12B02), and the Fok Ying-Tong Education Foundation for Young Teachers in the Higher Education Institutions of China (Grant No. 121049).

\section{REFERENCES}

[1] C. Xu, Z. Horita and T. G. Langdon, "The Evolution of Homogeneity in an Aluminum Alloy Processed Using High-Pressure Torsion," Acta Materialia, Vol. 56, No. 18, 2008, pp. 5168-5176. doi:10.1016/j.actamat.2008.06.036

[2] C. Xum Z. Horita and T. G. Langdon, "Evaluating the Influence of Pressure and Torsional Strain on Processing by High-Pressure Torsion," Journal of Materials Science, Vol. 43, No. 23-24, 2008, pp. 7286-7292. doi:10.1007/s10853-008-2624-z

[3] A. P. Zhilyaev, A. A. Gimazov, E. P. Soshnikova, Á. Révész and T. G. Langdon, "Microstructural Characteristics of Nickel Processed to Ultrahigh Strains by HighPressure Torsion," Materials Science and Engineering A, Vol. 489, No. 1-2, 2008, pp. 207-212.

doi:10.1016/j.msea.2007.12.031

[4] A. P. Zhilyaev, K. Oh-ishi, T. G. Langdon and T. R. McNelley, "Microstructural Evolution in Commercial Purity Aluminum during High Pressure Torsion," Materials Science and Engineering A, 2005, Vol. 410-411, pp. 277 280. doi:10.1016/j.msea.2005.08.044

[5] A. P. Zhilyaev and T. G. Langdon, "Using High-Pressure Torsion for Metal Processing: Fundamentals and Applications," Progress in Materials Science, Vol. 53, No. 6, 2008, pp. 893-979. doi:10.1016/j.pmatsci.2008.03.002

[6] N. Lugo. N. Llorca. J. M. Cabrera and Z. Horita, "Microstructures and Mechanical Properties of Pure Copper Deformed Severely by Equal-Channel Angular Pressing and High Pressure Torsion," Materials Science and Engineering: A, Vol. 477, No. 1-2, 2008, pp. 366-371. doi:10.1016/j.msea.2007.05.083

[7] L. Kurmanaeva. Y. Ivanisenko. J. Markmann. C. Kübel, A. Chuvilin. S. Doyle. R. Z. Valiev and H. J. Fecht, "Grain Refinement and Mechanical Properties in Ultrafine Grained Pd and Pd-Ag Alloys Produced by HPT," Materials Science and Engineering: A, Vol. 527, No. 7-8, 2010, pp. 1776-1783. doi:10.1016/j.msea.2009.11.001

[8] R. Z. Caliev, R. K. Islamgaliev and I. P. Semenova, "Superplasticity in Nanostructured Materials: New Challenges," Materials Science and Engineering A, Vol. 463, No. 1-2, 2007, pp. 2-7. doi:10.1016/j.msea.2006.08.121 
[9] H. S. Kim, W. S. Ryu, M. Janecek, S. C. Baik and Y. Estrin, "Effect of Equal Channel Angular Pressing on Microstructure and Mechanical Properties of IF Steel," Advanced Engineering Materials, 2005, Vol. 7, No. 1-2, pp. 43-45. doi:10.1002/adem.200400146

[10] B. Hadzima. M. Janecek. Y. Estrin and H. S. Kim, "Microstructure and Corrosion Properties of Ultrafine-Grained Interstitial Free Steel," Materials Science and Engineering A, Vol. 462, No. 1-2, 2007, pp. 243-247. doi:10.1016/j.msea.2005.11.081

[11] M. Kawasaki, B. Ahn and T. G. Langdon, "Effect of Strain Reversals on the Processing of High-Purity Aluminum by High-Pressure Torsion," Journal of Materials Science, Vol. 45, No. 17, 2010, pp. 4583-4593. doi:10.1007/s10853-010-4420-9

[12] M. Kawasaki, R. Figueiredo and T. G. Langdon, "An Investigation of Hardness Homogeneity throughout Disks Processed by High-Pressure Torsion," Acta Materialia, Vol. 59, No. 1, 2011, pp. 308-316. doi:10.1016/j.actamat.2010.09.034

[13] S. C. Yoon, A. V. Nagasekhar and H. S. Kim, "Finite Element Analysis of the Bending Behavior of a Workpiece in Equal Channel Angular Pressing," Metals and Materials International, Vol. 15, 2009, pp. 215-219. doi:10.1007/s12540-009-0215-4

[14] Y. Ivanisenko, W. Lojkowski, R. Z. Valiev and H. T. Fecht, "The Mechanism of Formation of Nanostructure and Dissolution of Cementite in a Pearlitic Steel during High Pressure Torsion," Acta Materials, Vol. 51, No. 18, 2003, pp. 5555-5570. doi:10.1016/S1359-6454(03)00419-1

[15] M. Kawasaki and T. G. Langdon, "The Significance of Strain Reversals during Processing by High-Pressure Torsion," Materials Science and Engineering A, Vol. 498, No. 1-2, 2008, pp. 341-348. doi:10.1016/j.msea.2008.08.021

[16] Y. P. Li, E. Onodera, H. Matsumoto and A. Chiba, " Correcting the Stress-Strain Curve in Hot Compression Process to High Strain Level," Metallurgical and Materials Transaction A, Vol. 40A, No. 4, 2009, pp. 982-990. doi:10.1007/s11661-009-9783-7

[17] D. Shahriari, A. Amiri and M. H. Sadeghi, "Study on Hot Ring Compression Test of Nimonic 115 Superalloy Using Experimental Observations and 3D FEM Simulation," Journal of Materials Engineering and Performance, Vol. 19, No. 5, 2010, pp. 633-642. doi:10.1007/s11665-009-9522-7 